\title{
Bottom-up soft-lithographic fabrication of three-dimensional multilayer polymer integrated optical microdevices
}

\author{
Yanyi Huang, ${ }^{\text {a) }}$ George T. Paloczi, Joyce K. S. Poon, and Amnon Yariv \\ Department of Applied Physics, Mail Code 128-95, California Institute of Technology, \\ Pasadena, California 91125
}

(Received 21 May 2004; accepted 5 August 2004)

\begin{abstract}
We develop a method to efficiently fabricate three-dimensional multilayer polymer microchips for integrated optical applications. This method uses soft lithography to mold the core structures on top of the cladding layers. By repeating the process, a three-dimensional multilayer integrated optical microdevice, which consists of several layers of individual planar optical devices, is fabricated. We demonstrate a dual-layer microring resonator optical filter device where the devices in the different layers show essentially identical transmission responses. (C) 2004 American Institute of Physics.
\end{abstract}

[DOI: $10.1063 / 1.1802380]$

Integrated optical devices, especially planar lightwave circuits (PLCs), are one of the important parts of modern communications and networking. Polymers, as one class of promising materials for the next generation of optical devices, have been investigated for decades. ${ }^{1}$ Through proper design and chemical synthesis, polymers show excellent potential for both passive and active media. ${ }^{2}$ Conventional PLCs are two-dimensional photonic circuits and the degree of integration is limited by the size of the devices. Stacking PLCs to make three-dimensional (3D) structures will efficiently increase the density of photonic circuits. Several polymer 3D integrated optical devices have been realized by conventional lithography. ${ }^{3,4}$ Recently, soft lithography ${ }^{5}$ has been applied to fabricate high quality polymer integrated optical devices. ${ }^{6-9}$ Moreover, soft lithography is also one of the powerful alternate fabrication methods to generate $3 \mathrm{D}$ multilayer structures. ${ }^{10,11}$

In this letter, we describe the development of a multistep soft lithography method to fabricate 3D multilayer integrated optical devices. The schematic fabrication procedure of the multistep soft lithography is shown in Fig. 1. A poly(dimethylsiloxane) (PDMS) stamp, which constitutes a negative relief of the device, is used as the mold to reproduce the polymeric core structure. It has been shown that soft lithography is efficient in reproducing polymer integrated optical devices with nanometer fidelity. ${ }^{7}$ The master device, from which the PDMS mold is taken, can be fabricated by any of a number of lithographic process. In our demonstration, electron beam lithography is used to generate the master structures of core layers. The master chip [Fig. 1(a)] is made of negative electron beam resist SU-8 (Microchem) and then the PDMS prepolymer (RTV-615 kit, GE) is poured on and cured [Fig. 1(b)]. After cooling down to room temperature, the cured PDMS layer can be easily peeled off [Fig. 1(c)] to serve as the soft mold. A polymer with a lower refractive index, such as OG-125 ( $n=1.46$, EpoTek), is spun on a silicon wafer and cured by UV light, serving as the lower cladding layer [Fig. 1(d)]. A drop of the solution of the core polymer with a higher refractive index is placed upon the cladding layer and then the PDMS mold is pressed on the substrate [Fig. 1(e)] until the solidification of the core is complete. By releasing

\footnotetext{
${ }^{\text {a)} E l e c t r o n i c ~ m a i l: ~ y a n y i @ c a l t e c h . e d u ~}$
}

the PDMS mold, the core structure, identical to the master device, is formed [Fig. 1(f)]. A layer of cladding material, is spun on top of the core layer just molded [Fig. 1(g)] and serves as both the upper-cladding layer of the first core layer underneath and the under-cladding layer of the second core layer on top. By repeating the molding-cladding procedure again [Figs. 1(h)-1(k)], a two-layer structure is fabricated. The thickness of the cladding layer between the two core layers can be adjusted by changing the parameters of spin coating and the concentration of the spin solution. By choosing the correct cladding material and film thickness, the crosstalk between core layers can be adjusted or avoided. This procedure can obviously be repeated to fabricate 3D multilayer structures with more than two layers.

There are several intrinsic advantages of applying the multistep soft lithographic method to fabricate integrated optical devices. First, compared with the other fabrication methods for 3D optical devices, such as multilayer grayscale optical lithography ${ }^{3}$ and two photon lithography, ${ }^{12}$ our multistep soft lithographic method has obvious cost implications. Furthermore, multistep soft lithography allows for the application of different core structures for the layers and integrating various optical functions in a single chip. As shown in Fig. 2(a), we fabricate a three-layer integrated optical microchip with a different structure in each layer. The lower layer (layer 1) is a waveguide Y splitter made of amorphous polycarbonate $(n=1.60)$. The molding solution is a dibromomethane solution containing $2 \mathrm{wt} \%$ polycarbonate (Aldrich). The middle layer (layer 2) is a ring resonator optical filter (ring diameter $400 \mu \mathrm{m}$ ) made of polystyrene ( $n$ $=1.60$ ). This core layer is molded from a 4 wt $\%$ toluene solution of polystyrene (Aldrich). The upper layer (layer 3) is a waveguide made of SU-8 $(n=1.57)$ by UV curing during the molding. The four cladding layers, between which the three different core layers are placed, are all made of OG125. This chip with three different core structures and different core materials can be fabricated by multistep soft lithography using only PDMS molds and a press, and each mold can be used hundreds of times for reproduction. With conventional etching techniques, this chip might require complicated and lengthy processing. This multistep procedure allows for the integration of different materials and devices on the same chip. Moreover, in contrast to conventional top- 


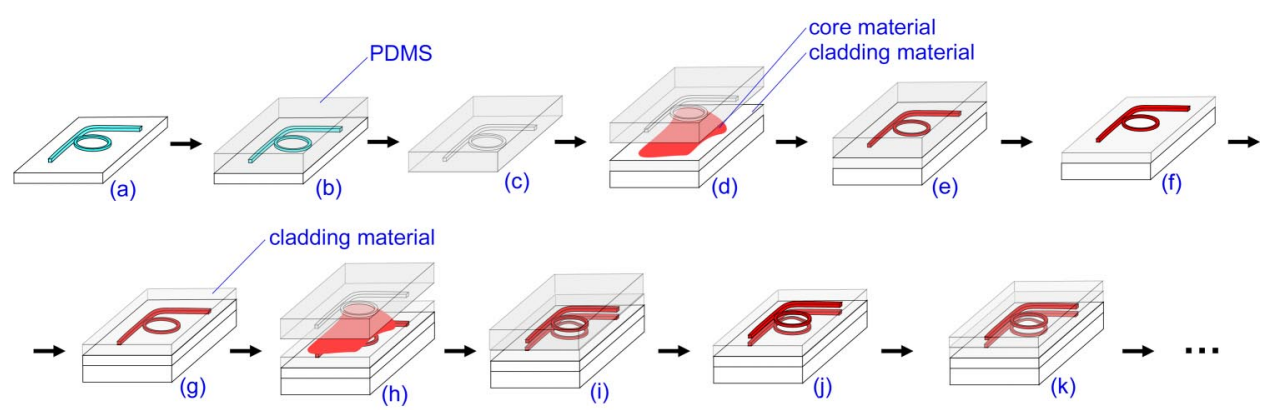

FIG. 1. (Color online) Schematic flowchart of the fabrication of 3D integrated optical microchips. down etching methods, the bottom-up method is more suitable for constructing 3D structures on existing devices, which may be important for hybrid optoelectronic integration.

In order to investigate the optical properties of each layer of the multilayer structure, we fabricate a two-layer microchip, in which both layers have microring resonator optical filters molded from the same PDMS mold. The microring resonator optical filter ${ }^{13,14}$ consists of a straight waveguide evanescently coupled to a filtering waveguide ring. When the coupling between the straight waveguide and the ring reaches the critical coupling condition, the resonance wavelengths will be completely attenuated at the output of the waveguide. Polymer microring resonator optical filter devices have been fabricated by different methods, including reactive ion etching, ${ }^{15}$ electron beam direct writing, ${ }^{16,17}$ nanoimprinting, ${ }^{18}$ and soft lithography. ${ }^{7,8}$

Figure 2(b) shows the optical microscope image of the 3D microchip containing two layers of microring resonator optical filters. Each optical filter has an input end and an
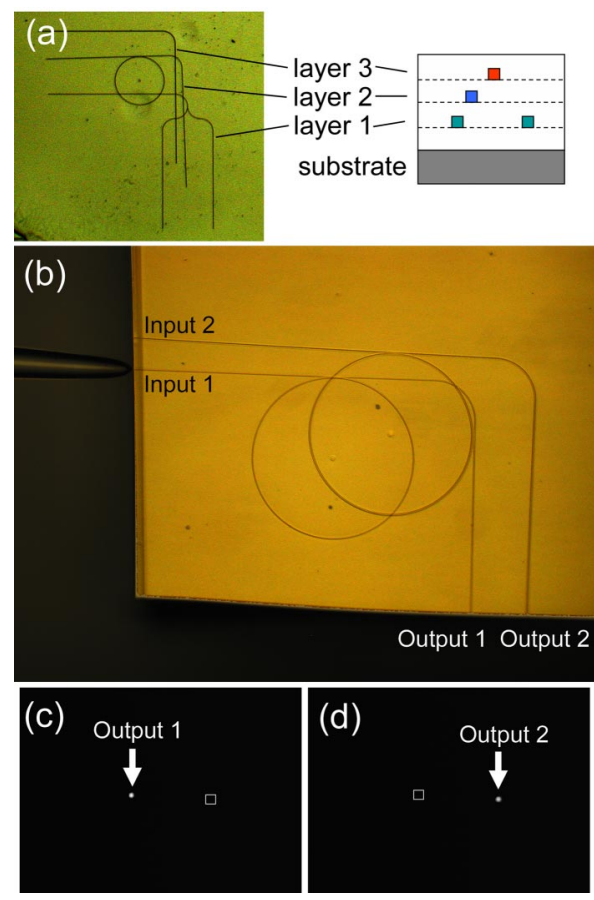

FIG. 2. (Color online) Optical microscope images of multilayer devices. A three-layer microchip and its schematic structure are shown in (a). Each layer consists of a different polymer material. A two-layer microchip containing a microring resonator optical filter in each layer is shown in (b); (c) and (d) are infrared camera images of the outputs of the microring resonator optical filters in the upper layer (input/output 1) and in lower layer (input/ output 2), respectively. The squares in (c) and (d) are the positions of end

facets of the other output.
Downloaded 14 Dec 2005 to 131.215 .225 .171 . Redistribution subject to AlP license or copyright, Downloaded 14 Dec 2005 to 131.215.225.171. Redistribution subject to AIP license or copyright, see http://apl.aip.org/apl/copyright.jsp output end [Input 1 and Output 1 in Fig. 2(b) belong to the upper layer while Input 2 and Output 2 the lower layer] and the end facets are prepared by cleaving the silicon substrate. The cores of the microring resonator optical filters are made from SU-8 and the cladding layers are OG-125. The cross section of the core waveguides is $2 \mu \mathrm{m} \times 1.6 \mu \mathrm{m}$ and the OG-125 layers are $3 \mu \mathrm{m}$ thick. Light from a tunable laser is coupled into one of the inputs through a tapered single-mode fiber and optical transmission from the outputs are collected by a $10 \times$ aspherical objective and then detected by an IR photodetector. The tapered fiber can be controlled by a moving stage to selectively couple the laser into one of the two inputs of the chip. When the laser is coupled into Input 1 (upper layer), we observe light at Output 1 [Fig. 2(c)]. When we move the tapered fiber to the lower layer and couple the laser into the Input 2, light is observed from Output 2 [Fig. 2(d)]. The position of the end facet of the waveguide in the layer that we have not coupled light into has been marked by a square in the images to indicate the horizontal and vertical displacement. Both images show the good mode confinement of the SU-8 waveguide surrounded by OG-125 cladding, indicating that the refractive index contrast is suitable for single mode transmission. Finite difference simulations confirm the waveguides are single-mode near $1550 \mathrm{~nm}$. No crosstalk is observed in the experiment due to the thick cladding layer between the core layers.

Figure 3 shows the transmission spectra of the dual-layer microring optical filter chip. In order to achieve the critical

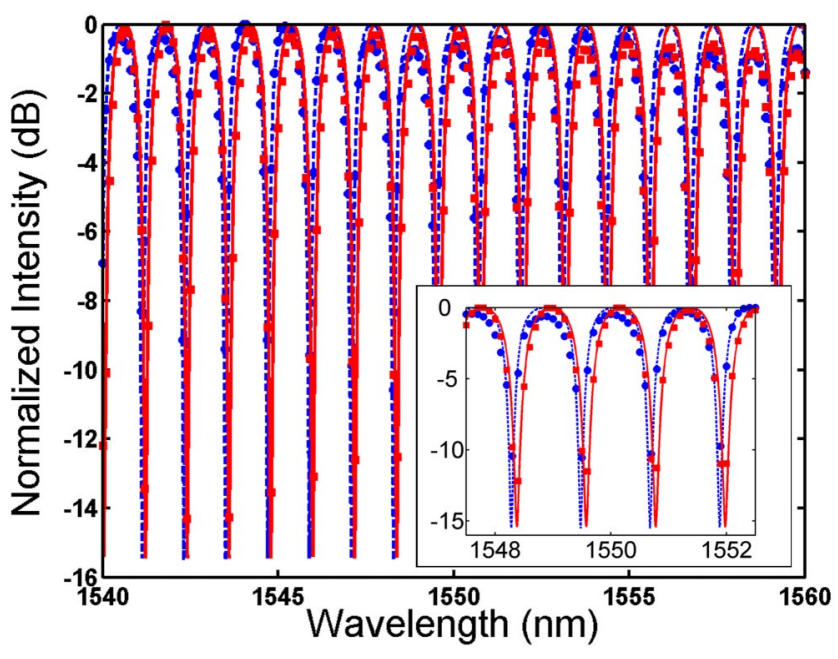

FIG. 3. (Color online). Transmission spectra of microring resonator optical filters in the two layers of the microchip [as shown in Fig. 2(b)]. The experimental data are shown as solid circle (lower layer) and solid square (upper layer). The solid line and dashed line are the fitting curves of experimental data of upper layer and lower layer, respectively. The inset shows the
detailed spectra near $1550 \mathrm{~nm}$. 
coupling condition, the gap between the straight waveguide and the ring resonator is designed to be $400 \mathrm{~nm}$. Both spectra show periodic notches with extinction ratios of around $-15 \mathrm{~dB}$ extinctions, indicating that the critical coupling condition is essentially achieved with our design. Besides the extinction ratio, which is extremely sensitive to the fabrication accuracy, the two spectra are also similar in terms of their free spectral ranges (FSRs) and shapes. The FSRs of the lower layer and upper layer are both measured to be $1.201 \mathrm{~nm}$. The unloaded quality $(Q)$ factors of these two microring resonators are estimated by data fitting to be around $1.0 \times 10^{4}$. The similarities indicate that the two layers of microring resonator optical filters are nearly identical. The $0.1 \mathrm{~nm}$ offset between the two spectra is due to slightly different effective refractive indices. According to the data fitting of the two spectra, the difference in the effective refractive indices is 0.0001 . The essentially identical microring resonator optical filters in this dual-layer device shows the significant accuracy and fidelity of soft lithography, as well as the potential of polymer integrated optical devices in $3 \mathrm{D}$ optical circuits.

In summary, our experimental demonstration of $3 \mathrm{D}$ multilayer integrated optical devices made by multistep soft lithography points the way to highly integrated multilayer optical circuits which can be easily fabricated with low cost polymer materials. Through a highly efficient and well controlled soft molding process, planar lightwave circuits with various functionalities in different materials can be stacked together to form a high density multifunctional optical chip.
Financial support from the National Science Foundation and Defense Advanced Research Projects Agency (Dr. D. Honey and Dr. R. Athale) is gratefully acknowledged.

${ }^{1}$ L. Eldada and L. W. Shacklette, IEEE J. Sel. Top. Quantum Electron. 6, 54 (2000).

${ }^{2}$ H. Ma, A. K.-Y. Jen, and L. R. Dalton, Adv. Mater. (Weinheim, Ger.) 14, 1339 (2002).

${ }^{3}$ S. M. Garner, S.-S. Lee, V. Chuyanov, A. Chen, A. Yacoubian, W. H. Steier, and L. R. Dalton, IEEE J. Quantum Electron. 35, 1146 (1999).

${ }^{4}$ J. S. Kim and J. J. Kim, J. Lightwave Technol. 22, 840 (2004).

${ }^{5}$ Y. Xia and G. M. Whitesides, Angew. Chem., Int. Ed. 37, 550 (1998).

${ }^{6}$ X.-M. Zhao, S. P. Smith, S. J. Waldman, G. M. Whitesides, and M. Prentiss, Appl. Phys. Lett. 71, 1017 (1997).

${ }^{7}$ Y. Huang, G. T. Paloczi, J. Scheuer, and A. Yariv, Opt. Express 11, 2452 (2003).

${ }^{8}$ Y. Huang, G. T. Paloczi, A. Yariv, C. Zhang, and L. R. Dalton, J. Phys. Chem. B 108, 8606 (2004).

${ }^{9}$ G. T. Paloczi, Y. Huang, A. Yariv, J. Luo, and A. K. Y. Jen, Appl. Phys. Lett. 85, 1662 (2004).

${ }^{10}$ L.-R. Bao, X. Cheng, X. D. Huang, L. J. Guo, S. W. Pang, and A. F. Yee, J. Vac. Sci. Technol. B 20, 2881 (2002).

${ }^{11}$ J. Zaumseil, M. A. Meitl, J. W. P. Hsu, B. R. Acharya, K. W. Baldwin, Y.-L. Loo, and J. A. Rogers, Nano Lett. 3, 1223 (2003).

${ }^{12}$ W. Zhou, S. M. Kuebler, K. L. Braun, T. Yu, J. K. Cammack, C. K. Ober, J. W. Perry, and S. R. Marder, Science 296, 1106 (2002).

${ }^{13}$ A. Yariv, Electron. Lett. 36, 321 (2000).

${ }^{14}$ A. Yariv, IEEE Photonics Technol. Lett. 14, 483 (2002).

${ }^{15}$ P. Rabiei, W. H. Steier, C. Zhang, and L. R. Dalton, J. Lightwave Technol. 20, 1968 (2002).

${ }^{16}$ G. T. Paloczi, Y. Huang, A. Yariv, and S. Mookherjea, Opt. Express 11, 2666 (2003)

${ }^{17}$ Y. Huang, G. T. Paloczi, J. K. S. Poon, and A. Yariv, Adv. Mater. (Weinheim, Ger.) 16, 44 (2004).

${ }^{18}$ C. Chao and L. J. Guo, J. Vac. Sci. Technol. B 20, 2862 (2002). 